\title{
Scalar mixing and strain dynamics methodologies for PIV/LIF measurements of vortex ring flows
}

Yann Bouremel and Andrea Ducci

Citation: Phys. Fluids 29, 013602 (2017); doi: 10.1063/1.4973822

View online: http://dx.doi.org/10.1063/1.4973822

View Table of Contents: http://aip.scitation.org/toc/phf/29/1

Published by the American Institute of Physics

\section{Articles you may be interested in}

Vortex identification from local properties of the vorticity field

Phys. Fluids 29, 015101015101 (2017); 10.1063/1.4973243

Effect of trailing edge shape on the separated flow characteristics around an airfoil at low Reynolds number:

A numerical study

Phys. Fluids 29, 014101014101 (2017); 10.1063/1.4973811

Effects of grid geometry on non-equilibrium dissipation in grid turbulence

Phys. Fluids 29, 015102015102 (2017); 10.1063/1.4973416

Modulating flow and aerodynamic characteristics of a square cylinder in crossflow using a rear jet injection

Phys. Fluids 29, 015103015103 (2017); 10.1063/1.4972982

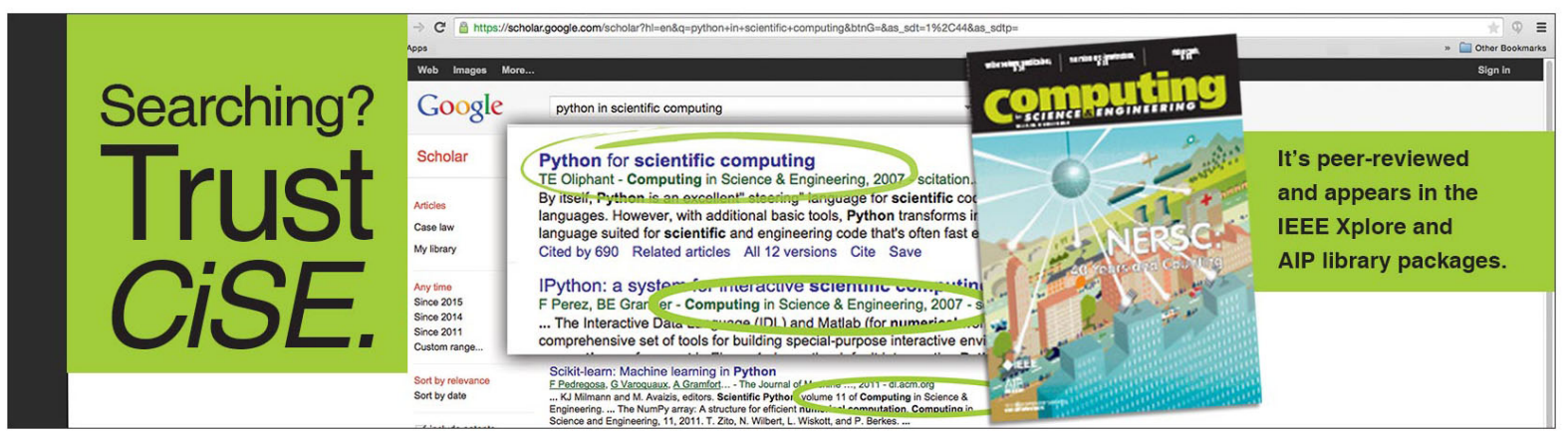




\title{
Scalar mixing and strain dynamics methodologies for PIV/LIF measurements of vortex ring flows
}

\author{
Yann Bouremel ${ }^{1,2, a)}$ and Andrea Ducci ${ }^{2}$ \\ ${ }^{1}$ NIHR Biomedical Research Centre at Moorfields Eye Hospital NHS Foundation Trust \\ and University College London, Institute of Ophthalmology, London, United Kingdom \\ ${ }^{2}$ Department of Mechanical Engineering, University College London, London, United Kingdom
}

(Received 16 August 2016; accepted 26 December 2016; published online 18 January 2017)

\begin{abstract}
Fluid mixing operations are central to possibly all chemical, petrochemical, and pharmaceutical industries either being related to biphasic blending in polymerisation processes, cell suspension for biopharmaceuticals production, and fractionation of complex oil mixtures. This work aims at providing a fundamental understanding of the mixing and stretching dynamics occurring in a reactor in the presence of a vortical structure, and the vortex ring was selected as a flow paradigm of vortices commonly encountered in stirred and shaken reactors in laminar flow conditions. High resolution laser induced fluorescence and particle imaging velocimetry measurements were carried out to fully resolve the flow dissipative scales and provide a complete data set to fully assess macro- and micro-mixing characteristics. The analysis builds upon the Lamb-Oseen vortex work of Meunier and Villermaux ["How vortices mix," J. Fluid Mech. 476, 213-222 (2003)] and the engulfment model of Baldyga and Bourne ["Simplification of micromixing calculations. I. Derivation and application of new model," Chem. Eng. J. 42, 83-92 (1989); "Simplification of micromixing calculations. II. New applications," ibid. 42, 93-101 (1989)] which are valid for diffusion-free conditions, and a comparison is made between three methodologies to assess mixing characteristics. The first method is commonly used in macro-mixing studies and is based on a control area analysis by estimating the variation in time of the concentration standard deviation, while the other two are formulated to provide an insight into local segregation dynamics, by either using an iso-concentration approach or an iso-concentration gradient approach to take into account diffusion. Published by AIP Publishing. [http://dx.doi.org/10.1063/1.4973822]
\end{abstract}

\section{INTRODUCTION}

Fluid mixing operations are central to possibly all chemical, petrochemical, and pharmaceutical industries either being related to biphasic blending in polymerisation processes, cell suspension for biopharmaceuticals production, and fractionation of complex oil mixtures. The stirred reactor configuration has historically been used for multi-phase mixing flows, with some geometry modifications being made to suit the specific process requirements. Recently novel reactor configurations have appeared, based on shaken or rocked motion, to meet the demand for gentler mixing which is required by new bio-therapeutics production processes. Up/down scaling from novel and traditional reactor configurations is still considered a challenging task due to the lack of deterministic equations and detailed fluid mechanics knowledge informing the translation. For example, shaken microwells are commonly used in parallel in bioprocess screening and development (Zhang et $a l .{ }^{4}$ ), but large-scale shaken bioreactors up to 10001 have been devised at the production level to reduce scaling problematics (Zhang et al. ${ }^{5}$ ), while in a similar strategy $\mathrm{ml}$ scale stirred reactors have entered the market.

Substantial efforts have been made in recent years to improve the understanding of the fluid mechanics of mixing

$\overline{{ }^{a)} \text { Electronic mail: y.bouremel@ucl.ac.uk }}$ at different spatial scales (i.e., macro-, meso-, and micromixing), encompassing both laminar and turbulent mixings, such understanding is still far from complete, and process optimisation often depends critically on process-specific data that are very useful, but generalisation and/or extrapolation of such knowledge to different vessel designs and scales is hampered by the complex dynamics involved. Traditional methodologies to assess macro-mixing are based on the variation in time of the concentration standard deviation or variance within the reactor (see the works of Busciglio et al. ${ }^{6}$ and Rodriguez et al. ${ }^{7}$ ). For example, the Coefficient of Variation, CoV, developed by Etchells and Meyer, ${ }^{8}$ is estimated from the concentration standard deviation normalised by the averaged steady state concentration, while Hartmann, Derksen, and van den Akker ${ }^{9}$ assessed the degree of mixing in stirred tank reactors by employing the volume weighted standard deviation of the following equation:

$$
c_{\text {mix }}=\sqrt{\left(\frac{\sum_{\tilde{i}}\left(\frac{c_{\tilde{i}}-\bar{c}}{\bar{c}}\right)^{2} \Delta V_{\tilde{i}}}{\sum_{\tilde{i}} \Delta V_{\tilde{i}}}\right)},
$$

with $\tilde{i}$ being the index of the monitoring point in the flow, $\Delta V_{\tilde{i}}$ the volume of the monitoring point $\tilde{i}$, and $\bar{c}$ being the average concentration in the total volume. Advanced mixing methodologies have been developed by Kukukova, Aubin, and Kresta, ${ }^{10,11}$ who defined a three-parameters based 
technique, using the intensity of segregation (directly linked to the coefficient of variation), the scale of segregation (based on iso-concentration), and the exposure to segregation reduction, and by Alberini et al., ${ }^{12,13}$ who defined the aerial distribution method, which captures the distribution of the percentage of the total area as a function of the level of mixedness, $X$ (directly linked to the coefficient of variation).

Advanced planar laser induced fluorescence, PLIF, measurements, based on inert and reactive dyes, are those of Kling and Mewes ${ }^{14}$ and Lehwald, Thévenin, and Zähringer ${ }^{15}$ to assess simultaneously macro- and micro-mixing in static mixers and stirred vessels, respectively. Kling and Mewes ${ }^{14}$ defined a local degree of micromixing, $\Delta c_{m}$, based on the reacted concentration $c_{2 r}(\boldsymbol{x}, t)$ and an hypothetical concentration, $c_{2}(\boldsymbol{x}, t)$, which would have been present in the same point if the reaction did not occur (see Equation (2)). The latter was estimated from the concentration of the inert dye, assuming that both were subject to the same macro-scale transport process,

$$
\Delta c_{m}(\boldsymbol{x}, t)=1-\frac{c_{2 r}(\boldsymbol{x}, t)}{c_{2}(\boldsymbol{x}, t)} .
$$

Models often employed to characterise mixing are the engulfment-deformation-diffusion model and its simplified version, termed the engulfment model which were developed by Baldyga and Bourne ${ }^{2,3}$ and are based on the deformation of inert/reacting dyes embedded in a turbulent flow under the assumption of negligible diffusion (Schmidt number, $S c=v / \kappa \ll 4000)$. A reactive or an inert blob of dye is stretched and thinned by the action of vortex tubes, of size around twelve Kolmogorov lengths, which entrain the surrounding fluid. It is assumed that on average each engulfment corresponds to an equal volume of fluid being entrained, with the volume growth rate of the eddies containing the inert tracer or reactant being proportional to the engulfment rate, $E_{e}=0.06 \sqrt{\epsilon / v}$ (see the following equation):

$$
\frac{d V_{e}}{d t}=E_{e} V_{e},
$$

with $\epsilon$ being the viscous dissipation rate of kinetic energy and $v$ the kinematic viscosity.

In view of the complexity of the mixing dynamics in stirred and shaken reactors, and the necessity of a reliable methodology to estimate the degree of mixing in the presence of a vortical structure, a more fundamental and simpler flow of a vortex ring was selected as a paradigm exhibiting similarities with the trailing vortex emanating from an impeller blade (i.e., Yianneskis, Popiolek, and Whitelaw ${ }^{16}$ ) and the toroidal vortices present in a shaken system (i.e., Weheliye, Yianneskis, and Ducci ${ }^{17}$ and Ducci and Weheliye ${ }^{18}$ ), as well as it provides a stirring motion similar to that of the engulfment model, where the interaction of strain and diffusion can be studied in detail.

Several works have investigated vortex rings and characterised its flow evolution through different stages, Refs. 19-26. Gharib, Rambod, and Shariff ${ }^{23}$ studied the effects of the stroke ratio $L / D$ on the formation process (where $L$ is the piston stroke and $D$ is the internal pipe exit diameter) and determined the range of stroke ratios, also denoted as the formation number, associated with the pinch-off stage. Their work covered several experimental conditions for stroke ratios in the range $0.4 \leq L / D \leq 14.5$, with varying piston velocity programs for impulsive, fast, and slow ramp piston motions, for different orifice diameters, and for varying tip angles $\left(20^{\circ}-90^{\circ}\right)$. Their work showed that for stroke ratios $L / D>4$ the vortex ring pinched off in a narrow range of piston displacements, $x / D \approx 4$, before the piston completed its stroke. This limit, $L / D=4$, corresponds to the maximum amount of energy that can be absorbed/stored by the vortex (i.e., the maximum vortex circulation possible) and to its maximum growth. For $L / D \leq 4$, pinch-off and piston stroke completion occurs at the same time. The pinch-off stage can also be considered as the moment when the velocity of the fluid delivered by the piston through the pipe is equal to the velocity of the vortex ring (Shusser et al. ${ }^{27}$ ).

The definitions of the vortex ring momentum, $I$, circulation, $\Gamma$, and Reynolds number, $R e$, are reported in Equations (4)-(6), respectively,

$$
\begin{aligned}
I & =A_{p} \rho L \overline{U_{p}}, \\
\Gamma & =\frac{1}{2} L \overline{U_{p}}, \\
R e & =\frac{\Gamma}{v},
\end{aligned}
$$

with $A_{p}$ being the pipe exit orifice area, $\rho$ the fluid volumetric mass, and $v$ the fluid kinematic viscosity. They are based on the slug-flow model, which assumes that the bulk flow velocity at the tube exit is the mean piston velocity $\left(\overline{U_{p}}\right)$, with a net energy transfer from the piston motion to the vortex ring flow (Shariff and Leonard ${ }^{28}$ ). It is worth noting that once the stroke reaches the pinch-off value, $L / D \approx 4$, ambient fluid is no longer entrained by the vortex ring as explained by Dabiri and Gharib. ${ }^{29}$ Once the roll-up process is completed, the vortex ring pinches off and moves forward under its self-induced velocity.

Shusser and Gharib ${ }^{30}$ defined the energy of a steady (pinched-off) vortex ring as follows:

$$
E_{v-r}=\beta \sqrt{\rho \Gamma^{3}}
$$

where $\beta$ is a non-dimensional energy parameter associated with the pinch-off process equal to $0.33 .{ }^{23}$ Mohseni and Gharib $^{24}$ found that the vortex ring translational velocity is equal to half of the piston velocity, $U_{p} / 2$, when the slug flow model is employed. This value has the same order of magnitude with the findings of Taylor ${ }^{31}$ for an ideal fluid $(v=0)$ where the vortex ring velocity was $0.436 U_{p}$.

The transport and stirring mechanisms during the formation stage of a vortex ring were experimentally and numerically investigated by Shadden, Lekien, and Marsden ${ }^{32}$ by means of Particle Imaging Velocimetry, PIV, and Lagrangian Coherent Structure, LCS, respectively. LCS is based on the distribution of finite-time Lyapunov exponents, which can be used to measure the stretching of a material point in time and identify the boundaries of vortical structures. ${ }^{32,33}$ LCS studies of Shadden et $a l .{ }^{33}$ showed that the volume of fluid entrained by the vortex increases linearly in time, while the stretching of three different vortex ring interfaces exhibited an exponential growth.

Cetegen and Aguirre ${ }^{34}$ analysed numerically the mixing of passive and chemically reactive scalars of a two 
dimensional vortex pair. The molecular mixing was characterised in terms of the probability density function of the scalar and from the coefficient of mixedness, $f$, defined in the following equation:

$$
f=\frac{4}{A_{v}} \int_{A_{v}} \frac{c}{c_{\max }}\left(1-\frac{c}{c_{\max }}\right) d A_{v},
$$

where $A_{v}$ is the area of the flow considered, and $c$ and $c_{\text {max }}$ are the local and maximum scalar concentrations, respectively. It should be noted that in this study, two scalars of the same volume were mixed and therefore complete molecular mixing is achieved when $\frac{c}{c_{\max }}=0.5$, where $f$ assumes values of 0 for no mixing and 1 for complete mixing. The results of Cetegen and Aguirre ${ }^{34}$ showed a linear evolution of the coefficient of mixedness, $f$, with non-dimensional time and vortex circulation, $\frac{\Gamma}{2 \pi v}$. Verzicco and Orlandi ${ }^{35}$ developed a new model to extend the applicability of the coefficient of mixedness, $f$, to conditions when the volume percentages of the two scalars being mixed are not the same. Their numerical results are in agreement with the ones obtained by Cetegen and Aguirre, ${ }^{34}$ but in this case the time-derivative of the mixedness was proportional to $\Gamma P e^{-0.33}$, where the Peclet number is the product of the Reynolds and Schmidt numbers $(P e=R e \times S c)$.

The experimental studies of Southerland et al. ${ }^{36}$ examined in more detail the molecular mixing processes occurring in the roll-up, pinch-off, and convection stages of the vortex ring. Their conclusions were based on the direct image visualisation of the scalar dissipation rate. They showed that when adjacent branches of the dye spiral along the vortex ring centreline come into contact, the scalar dissipation rate decreases. This trend was attributed to the increase of the diffusion term $-\frac{1}{R e S c} \frac{\partial}{\partial x_{j}}\left(\frac{\partial c}{\partial x_{i}}\right) \frac{\partial}{\partial x_{j}}\left(\frac{\partial c}{\partial x_{i}}\right)$ in the advection-diffusion equation of the scalar dissipation rate as shown in the following equation:

$$
\begin{aligned}
\left(\frac{\partial}{\partial t}+\right. & \left.u_{i} \frac{\partial}{\partial x_{i}}-\frac{1}{R e S c} \Delta\right) \frac{1}{2} \frac{\partial c}{\partial x_{i}} \frac{\partial c}{\partial x_{i}} \\
& =-\frac{\partial c}{\partial x_{j}} S_{i j} \frac{\partial c}{\partial x_{i}}-\frac{1}{R e S c} \frac{\partial}{\partial x_{j}}\left(\frac{\partial c}{\partial x_{i}}\right) \frac{\partial}{\partial x_{j}}\left(\frac{\partial c}{\partial x_{i}}\right)
\end{aligned}
$$

Rajes and Mahesh ${ }^{37}$ investigated numerically the mixing of a vortex ring as a function of $L / D$. Their results show that the fluid entrainment process occurring in the vortex ring has relevant implications for mixing: the mixing induced by a vortex ring with a trailing jet, for example, for $L / D=6$, is less effective in terms of fluid entrainment than that obtained by two vortex rings produced in sequence with $L / D=4$ and $L / D=2$, respectively. In other words, the trailing jet entrains a lower amount of ambient fluid (i.e., does not enhance mixing) than two vortex rings generated one after the other for the same total piston displacement. They also showed that vortex ring fluid entrainment/mixing increases linearly with $L / D$.

Olcay and Krueger ${ }^{38,39}$ carried out an experimental (PLIF, PIV) and numerical analysis of ambient fluid entrainment during vortex ring formation using LCS for a range of stroke ratios ( $L / D$ from 0.5 to 3 ) and velocity programs. They have shown that the stroke ratio $L / D$ has an effect on ambient fluid entrainment and momentum as an increase of $L / D$ from 0.5 to 2 leads to a fluid entrainment fraction decrease of $67 \%$. They also found that the piston velocity program affects the momentum and entrainment rate of ambient fluid as well as the momentum of ejected and added mass of the vortex ring formation. For example, they show that the time evolution of the entrainment rate can be increased by up to initially $50 \%$ when comparing fast initial acceleration such as trapezoidal profiles with slower initial accelerations such as triangular positive slope velocity programs. When looking at the same effect on deceleration, they were able to calculate a $10 \%$ increase in the entrainment rate time profile during that time period when comparing fast to slow deceleration.

Finally, Meunier and Villermaux ${ }^{1}$ developed a model to characterise the effect of shear rate on the mixing of a LambOseen vortex with the assumption of negligible diffusion. According to Meunier and Villermaux ${ }^{1}$ if a material line grows linearly, then its thickness decreases according to the following equation:

$$
\delta_{n}(t, r)=\frac{\delta_{n_{o}}}{\sqrt{1+\left(S_{n s} t\right)^{2}}},
$$

where $\delta_{n}(t, r)$ is the local thickness at time $t, \delta_{n_{o}}$ the thickness at the initial time, and $S_{n s}$ the local shear rate. They provide a starting point to analyse the deformation of dye in a vortex ring in terms of stretching/compression. More recently, Meunier and Villermaux ${ }^{40}$ introduced a numerical method called the diffusive strip intended for scalar mixing in two-dimensional advection fields when the thickness of the strip is smaller than its local curvature radius.

This work aims at informing mixing operations in chemical and biopharma industries by providing a fundamental understanding of the mixing and stretching dynamics occurring in a vortex ring flow, which has been selected here as a flow paradigm of the vortices commonly encountered in stirred and shaken reactors in laminar flow conditions. High resolution LIF and PIV measurements were carried out to fully resolve the flow dissipative scales and provide a complete data set to fully assess macro- and micro-mixing characteristics. The analysis builds upon the Lamb-Oseen vortex work of Meunier and Villermaux ${ }^{1}$ and the engulfment model of Baldyga and Bourne, ${ }^{2,3}$ which are valid for diffusion free conditions, and a comparison is made between three methodologies to assess mixing characteristics. The first method is the most commonly used in macro-mixing studies and is based on a control area analysis by estimating the variation in time of the concentration standard deviation, while the other two are formulated to provide an insight into local segregation dynamics, by either using an iso-concentration approach, similar to common segregation scale characterisation methodologies, or an iso-concentration gradient approach to take into account diffusion.

\section{VORTEX RING FLOW GENERATION AND PATTERN}

The vortex ring was produced with an impulsive acceleration that generates a constant piston velocity driven in a horizontal pipe of diameter $D=2.86 \mathrm{~cm}$, discharging into a quiescent chamber. The piston velocity and position profiles are shown in Figure 1(a) for $U_{\text {pmax }}=2.5 \mathrm{~cm} / \mathrm{s}$ and $L=2.86 \mathrm{~cm}$. The simultaneous PIV and LIF measurements included in this work were carried out at a $R e=357.5$ 


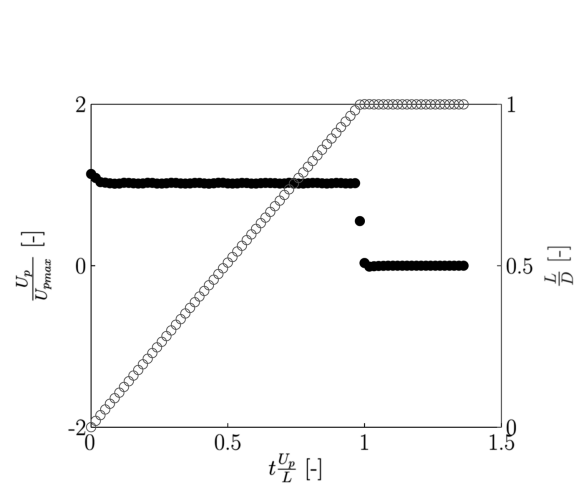

(a)

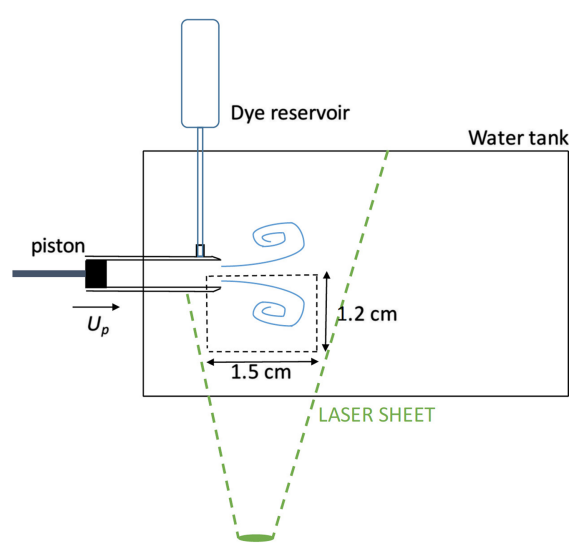

(c)

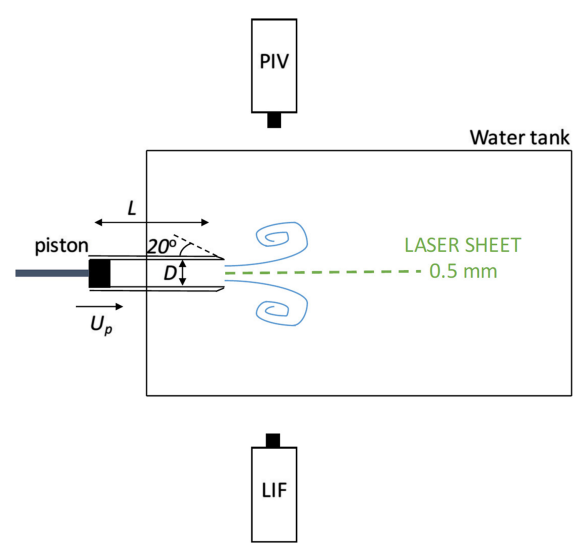

(b)

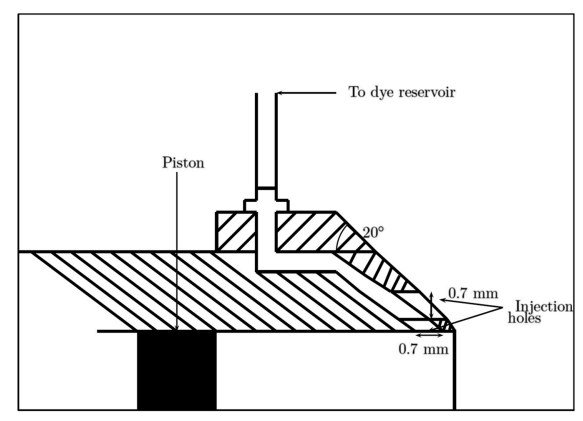

FIG. 1. (a) Variation of the piston velocity and position in time: $(\bullet)$ : $\frac{U_{p}}{U_{p \max }} ;\left(\right.$ (o): $\frac{x}{D}$; (b) top view, and (c) side view of the schematic experimental setup; (d) cross section of the pipe exit with details of the dye injection channels. The schematics are not drawn to scale. based on the slug flow model of Equation (6) (laminar vortex ring).

Rhodamine $6 \mathrm{G}$, at a concentration of $c_{\max }=7.4 \mathrm{mg} / \mathrm{l}$, was injected through 8 pairs of holes in the pipe exit. Its Schmidt number is $S c=v / \kappa=1280$, where $\kappa$ is the dye diffusion coefficient. Prior to the start of the experiment, a blob of dye was allowed to form by gravity right below the pipe exit, and it was then deformed and entrained in the vortex ring during the roll-up stage. Figures 1(b)-1(d) provide a schematic of the experimental setup of the top view, side view, and cross section of the pipe exit with details of the dye injection channels, respectively.

LIF and PIV measurements were carried out on a vertical laser sheet $0.5 \mathrm{~mm}$ thick passing through the pipe axis, with the two cameras being focused on the laser plane from opposite sides (see Figures 1(b) and 1(c)). The laser setup consists of a ND:YAG Lee laser, 800 series mounted with a laser pulse energy monitor that was constantly checked throughout the experiments to record pulse-to-pulse energy fluctuations, which can potentially affect concentration measurements. The analysis of the data downloaded from the laser monitor unit indicated that pulse-to-pulse fluctuations were less than $1 \%$. The seeding particles for PIV measurements were silver-coated hollow glass particles with a mean diameter of $10 \mu \mathrm{m}$, specifically designed for flow application (neutrally buoyant). The PIV camera mounted a $60 \mathrm{~mm}$ lens, with a field of view of $4.2 \mathrm{~cm} \times 3.4 \mathrm{~cm}$. The PIV interrogation cell was set to $32 \times 32$ pixels with a $50 \%$ overlap, so that each PIV snapshot comprised $79 \times 63$ velocity vectors, with a spatial resolution of $0.53 \mathrm{~mm}$. The LIF camera was equipped with a $105 \mathrm{~mm}$ lens and focussed on a smaller area of $1.5 \mathrm{~cm} \times 1.2 \mathrm{~cm}$. The LIF spatial resolution was equal to the size of one pixel, $12 \mu \mathrm{m}$, and each image comprised $1280 \times 1024$ pixels. Both cameras were 8 bit, which implies that LIF measurements were obtained with a 255 range of grey scales. Initial concentration, $c_{\text {max }}$, of the injected rhodamine was optimised to avoid camera saturation and at the same time take advantage of the full grey scale range. Based on this, the resolution of the measured concentration was $\mathrm{d} c \approx 0.004 c_{\max }$. PIV and LIF images were recorded with a frame rate of $1 \mathrm{kHz}$. It should be stressed that to obtain an optimal displacement of the seeding particles inside each interrogation cell, and therefore an accurate estimation of the local velocity field, pairs of PIV images with a $40 \mathrm{~ms}$ time lag were analysed with the PIV adaptive correlation routine.

Characteristic velocity vector maps and dye concentration contour plots are shown in Figures 2(a)-2(d) for four different instants during the vortex ring roll-up stage $\left(t=0.25 L / U_{p}-0.71 L / U_{p}\right)$. To maximise the spatial resolution of the LIF and PIV measurements and to resolve the flow/scalar dissipative scale, the investigation area was focused on the bottom part of the vortex ring. From Figure 2, it is evident that as the rolling-up proceeds, the length of the streak of dye embedded in the vortex ring increases, while its thickness decreases. 
(a)

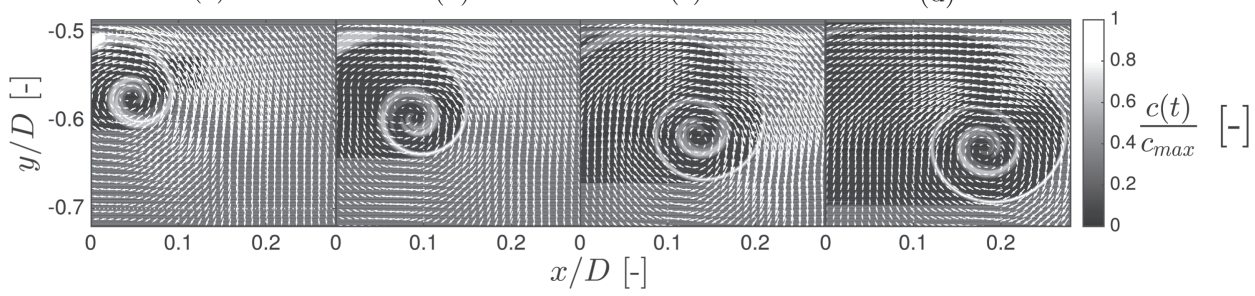

FIG. 2. Vortex ring velocity vector field and dye concentration contour plot at different times, (a) $t=0.25 \frac{L}{U_{p}} ;$ (b) $t=$ $0.41 \frac{L}{U_{p}} ;$ (c) $t=0.56 \frac{L}{U_{p}} ;$ (d) $t=0.71 \frac{L}{U_{p}}$.

\section{CONTROL AREA APPROACH}

In this section, the mixing efficiency of the vortex ring is investigated by adopting a control area approach applied to the rectangular area, $x / D=0-0.39$ and $y / D=0.41-0.84$, visualised by the dashed lines of Figure 3 . The spatial averages of the instantaneous mean, $\overline{c(t)}$, and standard deviation, $c_{s t d}(t)$, of the dye concentration within the control area are defined in Equations (11) and (12), respectively,

$$
\begin{gathered}
\overline{c(t)}=\frac{1}{N_{p}} \sum_{i=1}^{N_{p}} c_{i}(t), \\
c_{s t d}(t)=\sqrt{\frac{1}{N_{p}-1} \sum_{i=1}^{N_{p}}\left(c_{i}(t)-\overline{c(t)}\right)^{2}},
\end{gathered}
$$

with $c_{i}(t)$ being the concentration of the $i$ th pixel at time $t$, and $N_{p}$ the total number of pixels (959658) present in the control area. In the current experiment, the amount of dye injected through the pipe $(\approx 0.1 \mathrm{ml})$ was six orders of magnitude lower than the volume of fresh water present in the tank $(\approx 1001)$. This meant that at steady state the concentration is zero $\left(c / c_{\max }=0\right)$, and therefore the coefficient of mixedness, $f$, elaborated by Cetegen and Aguirre ${ }^{34}$ and Verzicco and Orlandi, ${ }^{35}$ which is valid for non-zero steady state concentration, could not be employed as an indicator of mixing. From this point of view, the spatially average standard deviation $c_{s t d}(t)$ is a valid alternative and is commonly used to assess mixing, with lower standard deviation being associated with a more uniform concentration distribution and a higher degree of mixing. The evolution in time of the mean dye concentration and of its standard deviation is shown in Figure 4. It can be seen that $\overline{c(t)}$ shows a sharp increase for $t \leq 0.48 \frac{L}{U_{p}}$ related to the increasing amount of dye entering the control

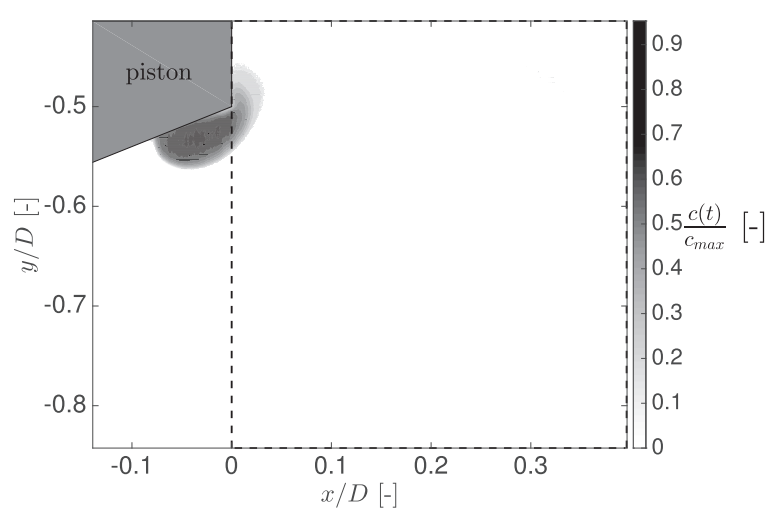

FIG. 3. Visualisation of the rectangular control area used to assess the mixing dynamics of the vortex ring. area, while a low decrease rate occurs for $t>\frac{L}{U_{p}}$, when part of the vortex ring starts exiting the control area. In the time range $t=[0.48-1] \frac{L}{U_{p}}, \overline{c(t)} / c_{\max }$ is characterised by a slight decrease, $4 \times 10^{-2}-3.5 \times 10^{-2}$, which is comparable in magnitude to the accuracy of the LIF system (i.e., $d c=0.004 c_{\max }$ ), which is visualised by the dashed lines $d c$ apart in Figure 4, and in first approximation the total amount of dye present in the control area during this period can be considered constant. Similarly to the study of Busciglio et al. ${ }^{6}$ in an unbaffled stirred tank reactor, which is characterised by a strong axial vortex, the standard deviation $c_{s t d}(t)$ follows an exponential decay, with a $17 \%$ reduction in the time range of nearly constant $\overline{c(t)}$, $t=[0.48-1] \frac{L}{U_{p}}$.

A better picture of the mixing evolution can be obtained from Figure 5 where the proportion of points, $\frac{N_{c}(t)}{N_{p}}$, associated with each concentration within the range of $c(t)$ examined, is shown at four different time instants, $t=0.25,0.4,0.55$, and $0.70 \frac{L}{U_{p}}$. The range of the ordinate axis has been limited to 0.2 to best visualise the variation of the concentration distribution over the highest end of the concentration range, but it is worth to stress that most of the fluid enclosed in the control volume area is associated with a nearly zero concentration (bulk fluid), and therefore these maxima $(\approx 80 \%)$ of the concentration distributions are not captured in Figure 5. From this point of view, a visual reference of their magnitude is provided by the vertical lines associated with the mean concentration, $\overline{c(t)}$, which is close to zero concentration for all the time instants considered. As mentioned before, the variation of $\overline{c(t)}$ is negligible when compared to the broad variation of the concentration distribution width, which is proportional to the concentration standard deviation, $c_{s t d}$. From

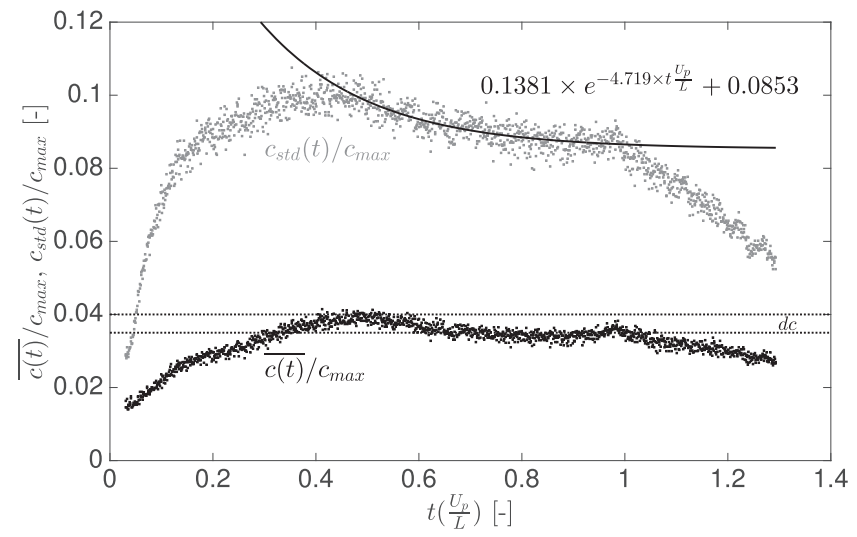

FIG. 4. Time evolution of the normalised mean dye concentration, $\overline{c(t)} / c_{\max }$, and non-dimensional standard deviation of the dye concentration, $c_{s t d}(t) / c_{\max }$. 


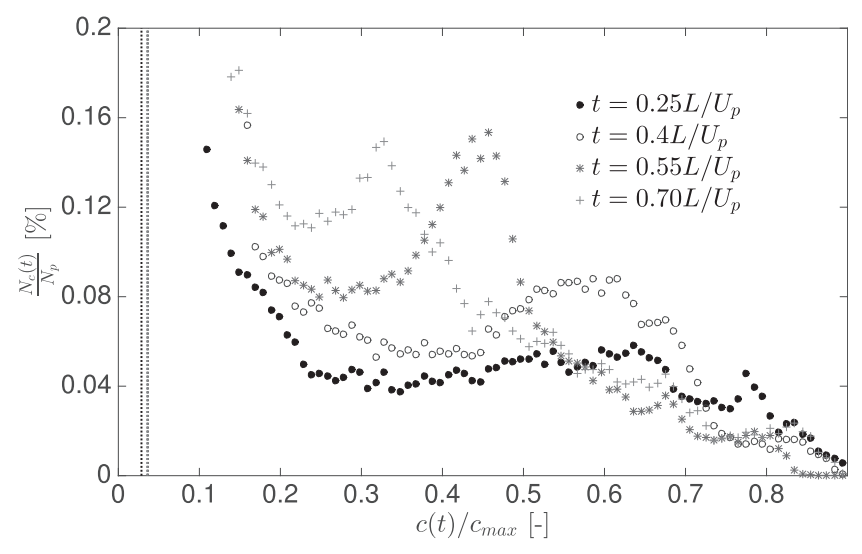

FIG. 5. Evolution of the percentage of points $\frac{N_{c}(t)}{N_{p}}$ with the concentration level, $c(t) / c_{\max }$, at four different times.

Figure $5, \frac{N_{c}(t)}{N_{p}}$ is characterised by a nearly uniform plateau over the concentration range $c / c_{\max }=0.2-0.6$ at $t=0.25 \frac{\mathrm{L}}{U_{p}}$, but as the experiment progresses the concentration distribution becomes bimodal, and beside the bulk fluid maximum at $c / c_{\max }$ $=0$, it starts to develop a second local peak, which is related to the high concentration dye entrained by the vortex ring. With increasing time, mixing takes place in proximity of the vortex border and the second peak increases in magnitude and tends to move towards the average concentration, $\bar{c}$, while its local distribution gets narrower, and in agreement to Figure 4 the overall standard deviation decreases.

\section{CONSERVATION OF A MATERIAL AREA}

In the second approach employed, the control area is defined by selecting a concentration threshold $\left(\frac{c}{c_{\max }}=0.3\right)$ and a local curvilinear reference system is employed, where $s$ and $n$ are local coordinates along and perpendicular to the centerline with origin at the vortex centre, and $\hat{s}(s)$ and $\hat{n}(s)$ are the corresponding unit vectors directed parallel and perpendicular to the local centreline direction, respectively. In this section, the thickness, $\delta_{n}(s, t)$, is defined as the distance between the inner and outer borders locally perpendicular to the centreline. Visualisation of $\hat{s}(s), \hat{n}(s)$, and $\delta_{n}(s, t)$ is provided in Figure 6 $\left(t=0.43 L / U_{p}\right)$. It is also worth noting that the dye embedded in the innermost part of the vortex core is characterised by a bifurcation, where a centreline cannot be unequivocally defined. As a consequence, this region, which accounts for only $2 \%$ of the total vortex ring area, was ignored during postprocessing. In the rest of the discussion, reference is made to "striation thickness" to indicate the thickness of a streak of dye, not the distance between adjacent runs of different fluids as often found in the literature (see Ottino ${ }^{41}$ ).

The variation in time of the growth ratio of the striation length, $\frac{\ell(t)-\ell_{o}}{\ell_{o}}$, is plotted in Figure 7(a), with $\ell_{o}$ corresponding to the centreline length at $t=0.078 \frac{L}{U_{p}}$. This was selected to be the start of the vortex ring development as for $t<0.078 \frac{L}{U_{p}}$, the shape of the vortex ring was not sufficiently developed to process the data and identify the striation centreline. It is noted that the centreline length increases linearly in time. The sudden drop of striation length occurring at $t=0.72 \frac{L}{U_{p}}$ is due to the

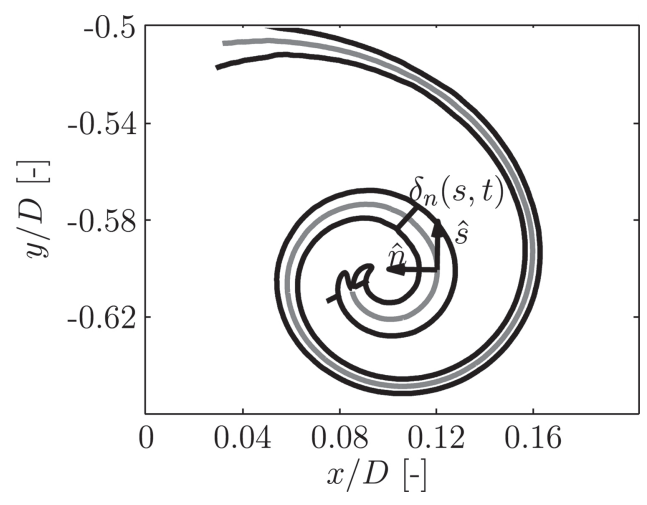

FIG. 6. Centreline identification at $t=0.43 \frac{L}{U_{p}}$ (grey line) with representation of the local curvilinear coordinate system $(\hat{s}, \hat{n})$. The black lines are obtained for $\frac{c}{c_{\max }}=0.3$.

vortex ring "pinch-off" associated with the iso-concentration contour selected $\left(\frac{c(t)}{c_{\max }}=0.3\right)$.

According to Meunier and Villermaux, ${ }^{1}$ when a material line grows linearly in time, its thickness decreases according to Equation (10). Figure 7(b) shows the variation in time of the spatially average thickness $\overline{\delta_{n}(t)}$, which is defined in

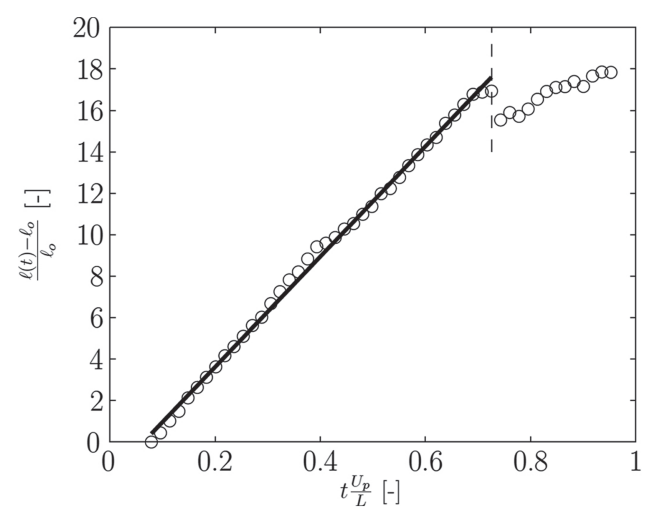

(a)

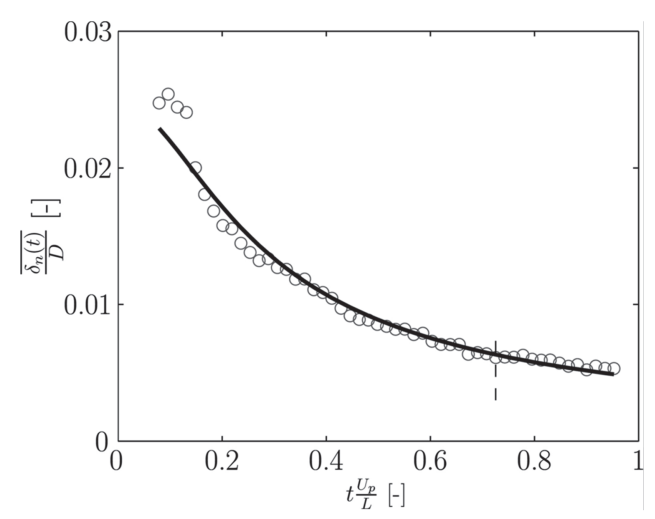

(b)

FIG. 7. (a) Evolution in time of the centreline growth ratio, $\frac{\ell(t)-\ell_{0}}{\ell_{0}}$; (b) variation in time of the dye thickness, $\frac{\overline{\delta_{n}(t)}}{D}$, spatially averaged along the centreline. The circular symbols are experimental data while the solid black line in (a) is the best fit line and in (b) is the curve from Equation (10) with $\left|S_{n s}\right|=5.1 \frac{U_{p}}{L}$. 
Equation (13) in both continuous and discrete forms,

$$
\overline{\delta_{n}(t)}=\frac{1}{\ell(t)} \int_{0}^{\ell} \delta_{n}(s, t) d s=\frac{1}{N(t)} \sum_{n=1}^{N(t)} \delta_{n}(s, t),
$$

where $N(t)$ is the total number of segments making up the centreline at time $t$. The decrease of $\frac{\overline{\delta_{n}(t)}}{D}$ with time in Figure 7(b) has been fitted with the inverse squared root function of Equation (10) which gave an average shear stress $\left|S_{n s}\right|$ $=5.1 \frac{U_{p}}{L}$. The contour level pinch-off time is denoted in Figure 7(b) as a dashed vertical line. Similar linear increase of material lines (proportional to $t$ ) have been reported in two dimensional incompressible flows by Meunier and Villermaux, ${ }^{1}$ for a Lamb-Oseen vortex and by Villermaux, ${ }^{42}$ where a linear increase of the length of a vortex is associated with an inverse law decrease of its thickness.

\section{CONSERVATION OF MATERIAL}

This section is concerned with the third, more general, approach where the diffusion process occurring during the vortex formation stage is taken into account by expanding/compressing the local region considered around the vortex centreline until the local concentration gradient has a predefined value. To compare with the engulfment model of Baldyga and Bourne, ${ }^{2,3}$ which assumes zero diffusion, the iso-concentration gradient delimiting the striation border was set to $|\partial \tilde{c}(t) / \partial n|<0.25$ greyscale pix $^{-1}$. The corresponding greyscale flux, $J$, across the border can be estimated from the following equation:

$$
J=-\kappa \frac{\partial \tilde{c}(t)}{\partial n} \times 2 \times \ell \times \frac{1}{\Delta x},
$$

where the border length is approximated to twice the vortex centreline length, $\ell, \kappa$ is the dye diffusion coefficient, and $\Delta x$ is the spatial resolution of the LIF measurements (i.e., $12 \mu \mathrm{m} / \mathrm{pixel}$ ). To put this in perspective, the greyscale flux across a striation $0.4 D$ long corresponds to $J=5 \times 10^{-6}$ greyscale $\mathrm{m}^{2} \mathrm{~s}^{-1}$, which over the $600 \mathrm{~ms}$ period considered in this experiment results in a concentration greyscale variation, $\Delta \tilde{c}=3 \times 10^{-6}$ greyscale $\mathrm{m}^{2}$. The corresponding total greyscale enclosed within the borders can be estimated according to the following equation:

$$
C=\int_{S} \tilde{c} d S=\sum_{i} \tilde{c}_{i} \times(\Delta x)^{2}=C_{t o t}(\Delta x)^{2}
$$

and for a striation $0.4 D$ long this corresponds to $C=10^{-4}$ greyscale $\mathrm{m}^{2}$. This means that only $3 \%$ of the total dye diffuses outside of the vortex border during the experiment $(\Delta \tilde{c} / C \approx 3 \%)$, and the assumption of the conservation of material and negligible diffusion is reasonable. This approach improves on the methodology outlined in Section IV, where the stretching of a material surface associated with a fixed concentration level of $\frac{c(t)}{c_{\max }}=0.3$ was considered regardless of the amount of material present within the borders (i.e., material could diffuse outside of the iso-concentration borders with different intensities along the centerline).

The contour plot of the dye concentration within the vortex ring at $t=0.74 \frac{L}{U_{p}}$ is shown in Figure 8(a), where blue and

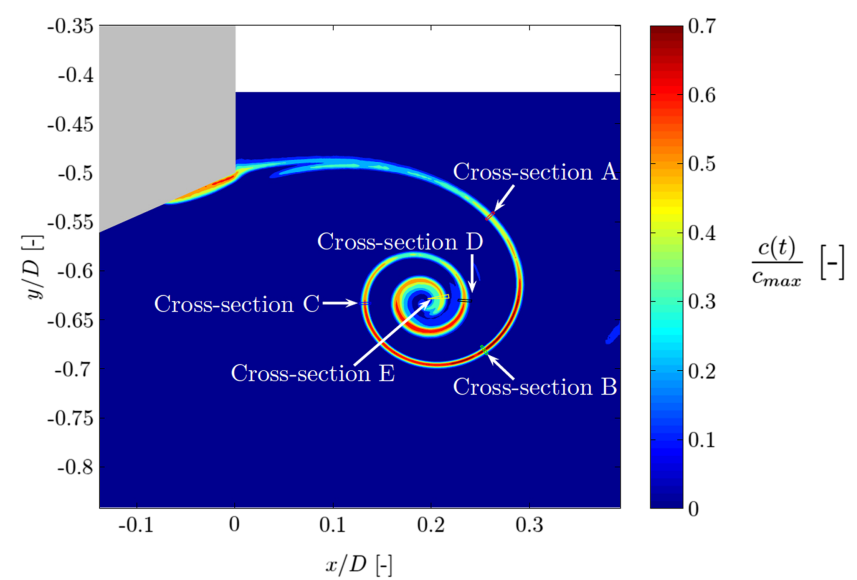

(a)

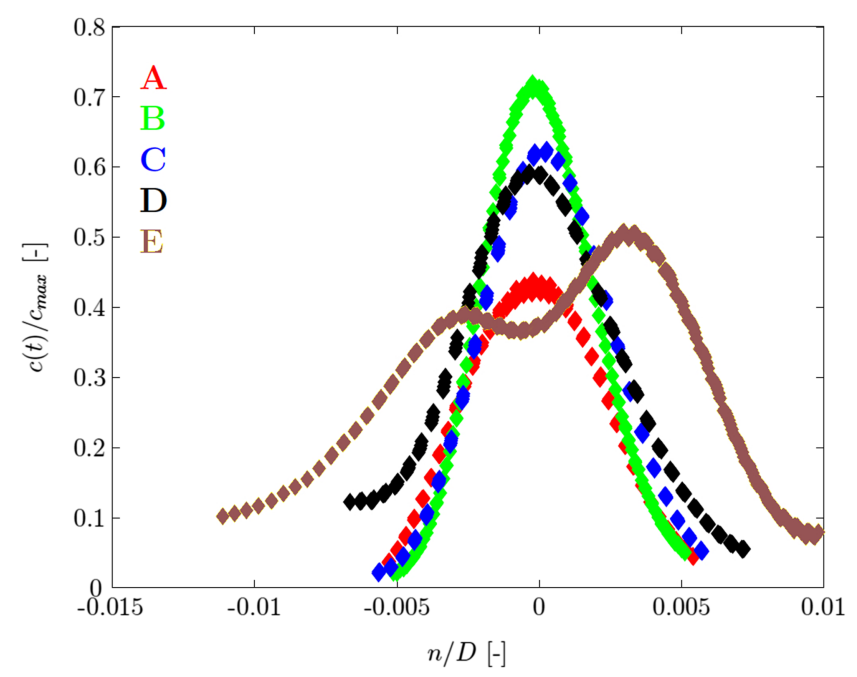

(b)

FIG. 8. (a) Contour plot of the dye concentration at $t=0.74 \frac{L}{U_{p}}$; (b) concentration profiles for 5 different cross sections along the vortex ring centreline.

red hues indicate low and high concentration values, respectively. Five concentration profiles at different cross sections locally orthogonal to the centreline are shown in Figure 8(b), where color-coded profiles are consistent with the cross section lines of Figure 8(a). Each point in the profile corresponds to a point within the particular cross section, where $\delta_{n}(s, t)$ is its distance from the local centreline. Four of the concentration profiles are denoted by a Gaussian shape centered along the centreline of the vortex ring, where the local concentration is a maximum. The brown profile associated with the innermost cross section considered shows two local maxima, in proximity of the centreline, which is reflected in the contour plot of Figure 8(a) with the bifurcation already outlined in Section IV. It should be noted that patches of high concentration are nonuniformly distributed along the centreline, which is due to the non-uniform suction of the dye during the roll-up process.

In Figure 9, the total amount of material considered, $C_{\text {tot }}$, is plotted as a function of the centreline length, $\ell$, for 8 time instants in the range $0.3 \frac{L}{U_{p}} \leq t \leq 0.92 \frac{L}{U_{p}}$. This plot allows to better visualise the growth of the striation, when different amounts of material are considered (i.e., $C_{t o t}$ ). As expected, the length of the centreline increases monotonically 


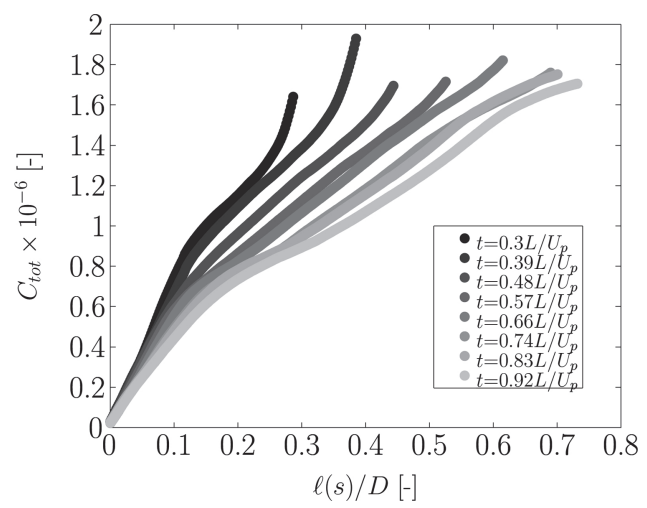

FIG. 9. Variation of the integral of the concentration, $C_{t o t}$, with the nondimensional length of the dye centreline, $\ell(s) / D$, at 8 different times.

as larger amounts of material are considered. For example, at $t=0.3 L / U_{p}$ total material values of $C_{\text {tot }}=0.8 \times 10^{-6}$ and $C_{\text {tot }}=1 \times 10^{-6}$ are spread over lengths of $\ell / D \approx 0.1$ and $\ell / D \approx 0.16$, respectively. From Figure 9 , it is evident that for each time instant the $C_{\text {tot }}$ plot can be subdivided into two $l$-ranges, with $C_{t o t}$ being denoted by very different material growth (i.e., local slope of the plot) for $\ell<0.1$ and $\ell>0.1$. For example, at $t=0.3 L / U_{p}$ the slope of $C_{\text {tot }}$ is approximately 8 for $\ell<0.1$ and 3.5 for $\ell>0.1$. Similarly, at $t=0.82 L / U_{p}$ it is around 4 for $\ell<0.1$ and 2 for $\ell>0.1$. This difference in material growth, which for $\ell<0.1$ is approximately double that for $\ell>0.1$, occurs for all the time instances and can be explained by considering that larger amounts of material are concentrated in the centre of the vortex core, while as the radial distance from the centre increases, the dye is spread over a longer distance. It is worth noting that at $t=0.3 L / U_{p}$ and $0.39 L / U_{p}$, the material growth is high also for large values of $\ell$, as in these cases, regions of the striation in close proximity to the injection point are considered.

To link the striation growth to the local stretching dynamics, the striation length was also estimated from the PIV data according to the local centreline increment reported in Equation (16), where $u_{n}$ and $u_{s}$ are the velocity components along the curvilinear reference system,

$$
\ell_{i}(t+d t)=\ell_{i}(t) \sqrt{\left(1+\frac{\partial u_{s}}{\partial s} d t\right)^{2}+\left(\frac{\partial u_{n}}{\partial s} d t\right)^{2}},
$$

with $\ell_{i}(t)$, the length of the $i$ th centreline segment at time $t$. The total centreline growth can be obtained by adding up the local segment increments as shown in the following equation:

$$
\Delta \ell(t+d t)=\sum_{i=1}^{N(t)}\left(\ell_{i}(t+d t)-\ell_{i}(t)\right)
$$

with $N(t)$, the total number of segments at time $t$. It should be noted that the number of segments varies in time and the centreline may comprise approximately up to 2000 segments. A comparison between the rates of growth of the vortex ring centreline length for $C_{t o t}=1 \times 10^{6}$ obtained from the LIF and the simultaneous LIF/PIV measurements based on Equation (17) is shown in Figure 10(a). The variation of the dye area with time for the same $C_{\text {tot }}$ value is shown in Figure 10(b). Figure 10(a) shows that the two methodologies are in good agreement, with

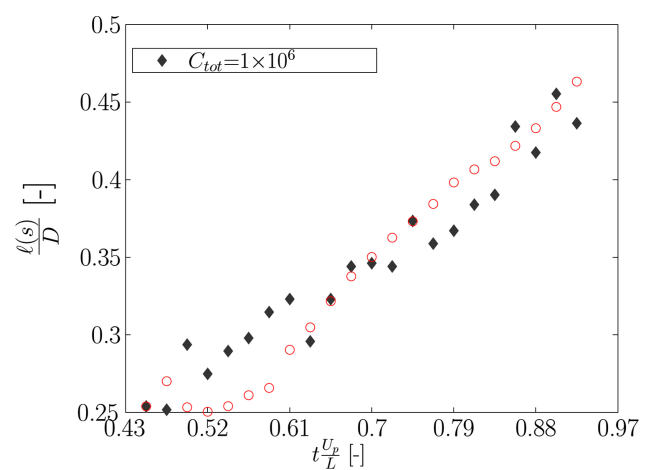

(a)

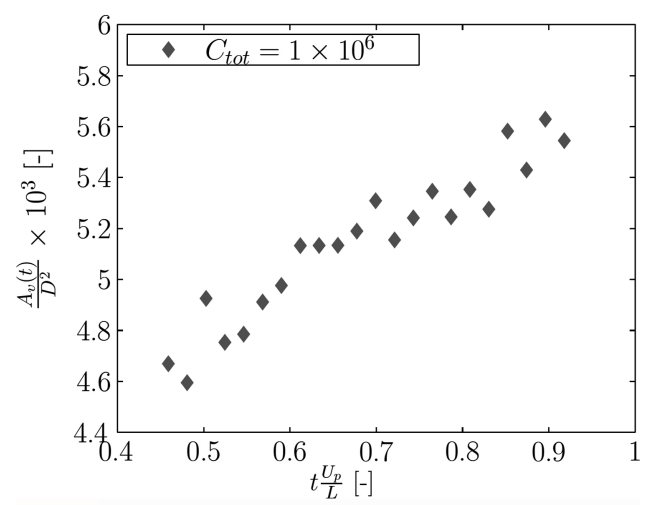

(b)

FIG. 10. (a) Variation of the non-dimensional centreline length, $\frac{\ell(s)}{D}$, with time for $C_{t o t}=1 \times 10^{6}:$ LIF data (black diamonds) and PIV/LIF method (red circles); (b) variation of the dye area, $\frac{A_{v}(t)}{D^{2}}$, with time for $C_{t o t}=1 \times 10^{6}$.

a maximum of $10 \%$ difference between the two figures. It is worth pointing out that in Figure 10(a) the centreline length increases by approximately $50 \%$ for $0.4 \leq U_{p} / L \leq 0.75$, while over the same time range the corresponding material area based on the iso-concentration approach of Figure 7 was denoted by an $80 \%$ increase. This implies that when the iso-concentration approach was used in Section IV, the striation length was estimated over different amounts of material at different time instances, and therefore was not an accurate representation of the material line growth. Similarly the material area, $A_{v}(t)$, associated with a constant $C_{t o t}$ of Figure 10(b) is denoted by a $20 \%$ increase, while when the iso-concentration approach was employed, the corresponding material area was approximately constant. This means that iso-concentration approaches, which are commonly used to assess segregation dynamics in mixing studies, should be employed with care as they are potentially affected by different degrees of diffusion along the striation length, and this discrepancy might become more relevant as the striation gets thinner and higher local gradients occur at the iso-concentration borders.

The local striation thickness, $\delta_{n}(s, t)$, is plotted in Figure 11(a) against the cumulative amount of dye, $C_{\text {tot }}$, for $t=0.31 L / U_{p}, 0.48 L / U_{p}, 0.65 L / U_{p}$, and $0.83 L / U_{p}$. The indicated variation with $C_{t o t}$ may be subdivided into four ranges of $C_{t o t}$ where the local thickness, $\delta_{n}(s, t)$, shows a different behaviour during the time interval examined. The range $0<C_{\text {tot }}<0.5 \times 10^{6}$ includes material which is closer to the vortex centre: in this region, the thinning dynamics is less 


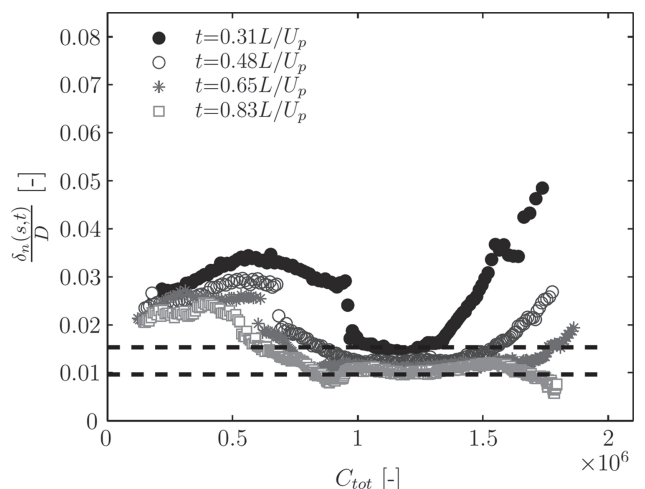

(a)

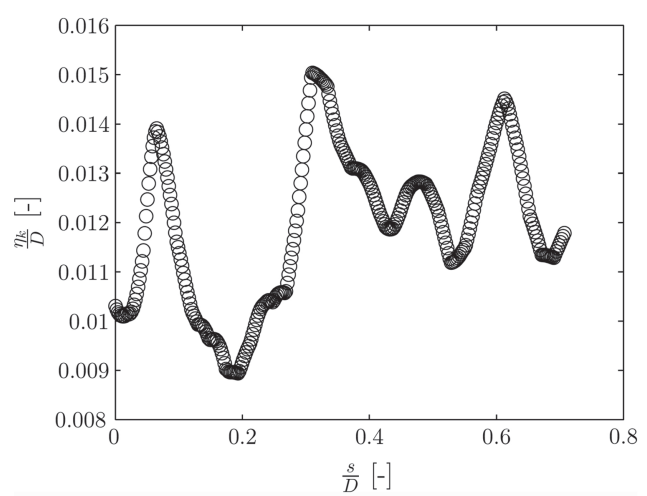

(b)

FIG. 11. (a) Variation of the non-dimensional thickness, $\frac{\delta_{n}(s, t)}{D}$, with the amount of concentration accumulated, $C_{t o t}$, along the dye centreline at 4 different times; the dashed lines show the local maximum and minimum value of the dissipative scale; (b) variation of the non-dimensional dissipative scale, $\frac{\eta_{k}}{D}$, with the non-dimensional curvilinear coordinate, $s / D$, at $t=0.65 \mathrm{~L} / \mathrm{Up}$.

significant as the variation in time of the local thickness is around $30 \%$, with a minimum thickness of around $0.02 \mathrm{D}$. The material included in the range $0.5 \times 10^{6} \leq C_{t o t} \leq 1 \times 10^{6}$ is subject to a higher degree of thinning, with nearly $70 \%$ reduction of the local striation thickness over the time interval considered. The material within $1 \times 10^{6} \leq C_{t o t} \leq 1.5 \times 10^{6}$ has already undergone a significant amount of thinning before the time interval reported $\left(t \leq 0.31 L / U_{p}\right)$ and its thickness has reached a lower limit of approximately $0.008 \mathrm{D}$. It is worth to point out that this corresponds to the smallest flow dissipative scale, $\eta_{k}$, associated with the rate of viscous dissipation of kinetic energy, $\epsilon$. Below $\eta_{k}$, mixing occurs solely by diffusion without any deformation action induced by the flow. The averaged maximum and minimum values obtained from the PIV measurements for $\eta_{k}$ are visualised as the two dashed lines in Figure 11(a) indicating that the PIV and LIF measurements reported in this work fully resolve all flow scales. Finally, the reduction of the local striation thickness for material between $1.5 \times 10^{6} \leq C_{t o t} \leq 2 \times 10^{6}$ is reduced by approximately $80 \%$.

The variation of the dissipative scale, $\eta_{k}$, with the curvilinear coordinate, $s$, is shown in Figure 11(b) for $t=0.65 L / U_{p}$. The dissipative length scale shows a periodic variation with increasing curvilinear coordinate, $s$, determined by the fact that the dissipation rate of kinetic energy also varies along the vortex centreline. The upper, $0.0153 \mathrm{D}$, and lower, 0.0097 $D$, thresholds reported in Figure 11(a) were obtained from the averages of the maxima and minima over the time range $[0.25-0.94] \frac{L}{U_{p}}$.

\section{CONCLUSIONS}

Simultaneous high resolution LIF and PIV measurements were carried out to fully resolve the flow dissipative scales and provide a complete data set to fully assess macro- and micro-mixing characteristics. The analysis builds upon the Lamb-Oseen vortex work of Meunier and Villermaux ${ }^{1}$ and the engulfment model of Baldyga and Bourne, ${ }^{2,3}$ which are valid for diffusion-free conditions, and a comparison is made between three methodologies to assess mixing characteristics.

The first approach describes macro-mixing by calculating the evolution in time of the concentration standard deviation using a control area analysis. The standard deviation was used as an indicator of mixing, with the lower value being associated with more uniform concentration distribution and a higher degree of mixing. It was shown as corroborated by Busciglio et $a{ }^{6}{ }^{6}$ that the standard deviation evolution in time obeys an exponential law decay. This approach provided a global view of the mixing occurring in the entire field of view.

A deeper understanding of the stretching/compression dynamics of the dye striation was obtained by determining the local growth of the striation length with the reduction of the local dye thickness. Two methodologies of increasing complexity were formulated to meet this task to give insight into the micro-mixing dynamics. The first method using an iso-concentration approach, similar to common segregation scale characterisation methodologies, aimed at investigating the variation in time of the striation geometry of a material area enclosed by the boundary of a pre-selected iso-contour level $\left(c(t) / c_{\max }=0.3\right)$, with the assumption of negligible diffusion at the contour borders. This method is dependent on the iso-contour level selected.

The second approach which is more refined is based on an iso-concentration gradient method and aimed at determining the variation in time of the striation characteristics associated with a constant amount of material, $C_{t o t}$. This allowed to take into account the diffusion process occurring during the vortex formation stage by expanding/compressing the local region considered around the vortex centreline until the local concentration gradient at the border could be considered negligible (i.e., $|\partial c(t) / \partial n|<0.25)$.

Both methodologies provided results consistent with those of Meunier and Villermaux ${ }^{1}$ and Villermaux, ${ }^{42}$ showing that a linear growth in time of the striation centreline was associated with an inverse law decrease of its thickness. The dye embedded in the vortex ring was associated with a streak that was thicker close to the vortex core and thinner further away.

The striation thickness determined from the LIF experiment was consistent with the PIV results, with the lowest values of $\delta_{n}(s, t)$ being limited by the local dissipative scale, which were directly estimated from the local instantaneous velocity gradients. The growth of the centreline length was estimated with different methodologies either by direct measurement from the LIF data or by integrating along the centreline the local stretching rates obtained from the PIV experiments. The results showed a good 
agreement with a maximum $10 \%$ difference between the two estimates.

It was shown that iso-concentration approaches, which are commonly used to assess segregation dynamics in mixing studies, should be employed with care as they are potentially affected by different degrees of diffusion along the striation length, and this discrepancy might become more relevant as the striation gets thinner and higher local gradients occur at the iso-concentration borders.

Future work is called for to apply and extend the proposed methodology to different operating conditions, including velocity programmes and higher $R e$, and assess to what extent local mixing dynamics are affected. This approach shows promise for the enhanced understanding of mixing and formulation of Lagrangian models and can provide meaningful results to obtain a better understanding of mixing dynamics in more complex flows such as those encountered in stirred tanks and shaken reactors.

\section{ACKNOWLEDGMENTS}

Financial support for the work reported here was provided by the Engineering and Physical Sciences Research Council (EPSRC) of the UK, Grant No. EP/D032539.

\section{NOMENCLATURE}

\section{Abbreviations}

$\mathrm{CoV}$ Coefficient of Variation

LCS Lagrangian Coherent Structure

LIF Laser Induced Fluorescence

PIV Particle Image Velocimetry

PLIF Planar Laser Induced Fluorescence

\section{Greek symbols}

$\beta \quad$ Non-dimensional energy parameter (-)

$\Gamma \quad$ Vortex ring circulation $\left(\mathrm{L}^{2} \mathrm{~T}^{-1}\right)$

$\delta_{n} \quad$ Thickness striation (L)

$\overline{\delta_{n}} \quad$ Spatial average of the thickness striation (L)

$\delta_{n_{i}} \quad$ Striation thickness associated to the $i$ th pair of adjacent points along the centreline $(\mathrm{L})$

$\delta_{n_{o}} \quad$ Thickness striation at the initial time (L)

$\Delta \tilde{c} \quad$ concentration greyscale variation $\left(\mathrm{L}^{2}\right)$

$\Delta c_{m} \quad$ Local degree of micromixing (-)

$\Delta l \quad$ Length incremental of the vortex ring centreline (L)

$\Delta V_{\tilde{i}} \quad$ Volume of the monitoring point $i\left(\mathrm{~L}^{3}\right)$

$\Delta x \quad$ Spatial resolution (L)

$\epsilon \quad$ Viscous dissipation rate of kinetic energy $\left(\mathrm{L}^{2} \mathrm{~T}^{-3}\right)$

$\kappa \quad$ Diffusion coefficient $\left(\mathrm{L}^{2} \mathrm{~T}^{-1}\right)$

$v \quad$ Kinematic viscosity $\left(\mathrm{L}^{2} \mathrm{~T}^{-1}\right)$

$\rho \quad$ Volumic mass $\left(\mathrm{ML}^{-3}\right)$

\section{Roman symbols}

$A_{p} \quad$ Pipe exit orifice area $\left(\mathrm{L}^{2}\right)$

$A_{v} \quad$ Dye area $\left(\mathrm{L}^{2}\right)$

$c$ Concentration $\left(\mathrm{ML}^{-3}\right)$

$\tilde{c} \quad$ Concentration in greyscale (-)

$\bar{c} \quad$ Spatial average of the concentration $\left(\mathrm{ML}^{-3}\right)$ $c_{i} \quad$ Concentration of the $i$ th pixel $\left(\mathrm{ML}^{-3}\right)$

$\tilde{c}_{i} \quad$ Concentration of the $i$ th pixel in greyscale (-)

$c_{\tilde{i}} \quad$ Concentration of the $i$ th monitoring point of the flow $\left(\mathrm{ML}^{-3}\right)$

$c_{\max }$ Maximum concentration $\left(\mathrm{ML}^{-3}\right)$

$c_{m i x}$ Volume weighted standard deviation of the concentration $\left(\mathrm{ML}^{-3}\right)$

$c_{\text {std }} \quad$ Standard deviation of the concentration $\left(\mathrm{ML}^{-3}\right)$

$C$ Surface integral of the greyscale concentration $\left(\mathrm{L}^{2}\right)$

$C_{\text {tot }}$ Total amount of material in greyscale (-)

$c_{2} \quad$ Hypothetical concentration $\left(\mathrm{ML}^{-3}\right)$

$c_{2 r} \quad$ Reacted concentration $\left(\mathrm{ML}^{-3}\right)$

D Piston diameter (L)

dc Resolution of the concentration $\left(\mathrm{ML}^{-3}\right)$

$E_{e} \quad$ Engulfment rate $\left(\mathrm{T}^{-1}\right)$

$E_{v-r} \quad$ Vortex ring energy $\left(\mathrm{ML}^{2} \mathrm{~T}^{-2}\right)$

$f \quad$ Mixedness coefficient (-)

$I \quad$ Vortex ring momentum $\left(\mathrm{MLT}^{-1}\right)$

$J \quad$ Greyscale flux $\left(\mathrm{L}^{2} \mathrm{~T}^{-1}\right)$

$\ell \quad$ Centreline length $(\mathrm{L})$

$\ell_{o} \quad$ Centreline length at $t=0.078 \frac{L}{U_{p}}(\mathrm{~L})$

$\ell_{i} \quad$ Length associated with the $i$ th pair of adjacent points along the centreline (L)

$L \quad$ Piston stroke (L)

$\hat{n} \quad$ Unit vector perpendicular to the local vortex ring centreline direction $(-)$

$N \quad$ Number of segments

$N_{c} \quad$ Number of pixels for a given concentration (-)

$N_{p} \quad$ Number of pixels (-)

$P e \quad$ Peclet number $(-)$

Re Reynolds number (-)

$\hat{s} \quad$ Unit vector parallel to the local vortex ring centreline direction (-)

Sc Schmidt number (-)

$S_{i j} \quad$ Strain rate tensor $\left(T^{-1}\right)$

$T_{m} \quad$ Mixing time (T)

$u_{i} \quad$ Flow velocity $\left(\mathrm{LT}^{-1}\right)$

$U_{p} \quad$ Piston velocity $\left(\mathrm{LT}^{-1}\right)$

$\overline{U_{p}} \quad$ Mean piston velocity $\left(\mathrm{LT}^{-1}\right)$

$U_{\text {pmax }}$ Maximum piston velocity $\left(\mathrm{LT}^{-1}\right)$

$V_{e} \quad$ Eddy volume $\left(\mathrm{L}^{3}\right)$

$x, y \quad$ Two-dimensional coordinate (L)

${ }^{1}$ P. Meunier and E. Villermaux, "How vortices mix," J. Fluid Mech. 476, 213-222 (2003).

${ }^{2}$ J. Baldyga and J. R. Bourne, "Simplification of micromixing calculations. I. Derivation and application of new model," Chem. Eng. J. 42, 83-92 (1989).

${ }^{3}$ J. Baldyga and J. R. Bourne, "Simplification of micromixing calculations. II. New applications," Chem. Eng. J. 42, 93-101 (1989).

${ }^{4}$ H. Zhang, S. R. Lamping, S. C. R. Pickering, G. J. Lye, and P. A. Shamlou, "Engineering characterisation of a single well from 24-well and 96-well microtitre plates," Biochem. Eng. J. 40, 138-149 (2008).

${ }^{5}$ X. Zhang, C. A. Bürki, M. Stettler, D. De Sanctis, M. Perrone, M. Discacciati, N. Parolini, M. DeJesus, D. L. Hacker, A. Quarteroni, and F. M. Wurm, "Efficient oxygen transfer by surface aeration in shaken cylindrical containers for mammalian cell cultivation at volumetric scales up to 1000 L,” Biochem. Eng. J. 45, 41-47 (2009).

${ }^{6}$ A. Busciglio, F. Grisafi, F. Scargiali, and A. Brucato, "Mixing dynamics in uncovered unbaffled stirred tanks," Chem. Eng. J. 254, 210-219 (2014).

${ }^{7}$ G. Rodriguez, T. Anderlei, M. Micheletti, M. Yianneskis, and A. Ducci, "On the measurement and scaling of mixing time in orbitally shaken bioreactors," Biochem. Eng. J. 82, 10-21 (2014). 
${ }^{8}$ A. Etchells III and C. Meyer, "Mixing in pipelines," in Handbook of Industrial Mixing: Science and Practice, edited by E. L. Paul, V. A. Atiemo-Obeng, and S. M. Kresta (John Wiley \& Sons, 2004), Chap. 7, pp. 391-478.

${ }^{9}$ H. Hartmann, J. J. Derksen, and H. van den Akker, "Mixing times in a turbulent stirred tank by means of LES," AIChE J. 52, 3696-3706 (2006).

${ }^{10}$ A. Kukukova, J. Aubin, and S. M. Kresta, "A new definition of mixing and segregation: Three dimensions of a key process variable," Chem. Eng. Res. Des. 87, 633-647 (2009).

${ }^{11}$ A. Kukukova, J. Aubin, and S. M. Kresta, "Measuring the scale of segregation in mixing data," Can. J. Chem. Eng. 89, 1122-1138 (2011).

${ }^{12}$ F. Alberini, M. J. H. Simmons, A. Ingram, and E. H. Stitt, "Assessment of different methods of analysis to characterise the mixing of shear-thinning fluids in a Kenics KM static mixer using PLIF," Chem. Eng. Sci. 112, 152169 (2014).

${ }^{13}$ F. Alberini, M. J. H. Simmons, A. Ingram, and E. H. Stitt, "Use of an areal distribution of mixing intensity to describe blending of non-Newtonian fluids in a Kenics KM static mixer using PLIF," AIChE J. 60, 332-342 (2014).

${ }^{14} \mathrm{~K}$. Kling and D. Mewes, "Two-colour laser induced fluorescence for the quantification of micro- and macromixing in stirred vessels," Chem. Eng. Sci. 59, 1523-1528 (2004).

${ }^{15}$ A. Lehwald, D. Thévenin, and K. Zähringer, "Quantifying macro-mixing and micro-mixing in a static mixer using two-tracer laser-induced fluorescence," Exp. Fluids 48, 823-836 (2010).

${ }^{16}$ M. Yianneskis, Z. Popiolek, and J. H. Whitelaw, "An experimental study of the steady and unsteady flow characteristics of stirred reactors," J. Fluid Mech. 175, 537-555 (1987).

${ }^{17}$ W. Weheliye, M. Yianneskis, and A. Ducci, "On the fluid dynamics of shaken bioreactors- flow characterization and transition," AIChE J. 59, 334-344 (2013).

${ }^{18}$ A. Ducci and W. H. Weheliye, "Orbitally shaken bioreactors-viscosity effects on flow characteristics," AIChE J. 60, 3951-3968 (2014).

${ }^{19}$ T. Maxworthy, "Some experimental studies of vortex rings," J. Fluid Mech. 81, 465-495 (1977).

${ }^{20}$ N. Didden, "On the formation of vortex rings: Rolling-up and production of circulation," J. Appl. Mech. Phys. 30, 101-116 (1979).

${ }^{21}$ D. I. Pullin, "Vortex ring formation at tube and orifice openings," Phys. Fluids 22, 401-403 (1979).

${ }^{22}$ T. T. Lim and T. B. Nickels, "Vortex rings," in Fluid Vortices, edited by S. I. Green (Kluwer Academic Publishers, Dordrecht, 1995).

${ }^{23}$ M. Gharib, E. Rambod, and K. Shariff, "A universal time scale for vortex ring formation,” J. Fluid Mech. 360, 121-140 (1998).
${ }^{24}$ K. Mohseni and M. Gharib, "A model for universal time scale of vortex ring formation,” Phys. Fluids 10, 2436-2438 (1998).

${ }^{25}$ J. J. Allen and B. Auvity, "Interaction of a vortex ring with a piston vortex," J. Fluid Mech. 465, 353-378 (2002).

${ }^{26}$ P. S. Krueger and M. Gharib, "The significance of vortex ring formation to the impulse and thrust of a starting jet," Phys. Fluids 15, 1271-1281 (2003).

${ }^{27}$ M. Shusser, M. Rosenfeld, J. Dabiri, and M. Gharib, "Effect of timedependent piston velocity program on vortex ring formation in a piston/cylinder arrangement," Phys. Fluids 18, 033601 (2006).

${ }^{28} \mathrm{~K}$. Shariff and A. Leonard, "Vortex rings," Annu. Rev. Fluid Mech. 24, 235-272 (1992).

${ }^{29}$ J. O. Dabiri and M. Gharib, "Fluid entrainment by isolated vortex rings," J. Fluid Mech. 511, 311-331 (2004).

${ }^{30}$ M. Shusser and M. Gharib, "Energy and velocity of a forming velocity ring," Phys. Fluids 12, 618-621 (2000).

${ }^{31}$ G. I. Taylor, "Formation of a vortex ring by giving an impulse to a circular disk and then dissolving it away," J. Appl. Phys. 24, 104 (1953).

${ }^{32}$ S. C. Shadden, F. Lekien, and J. E. Marsden, "Definition and properties of Lagrangian coherent structures from finite-time Lyapunov exponents in two-dimensional aperiodic flows," Physica D 212, 271-304 (2005).

${ }^{33}$ S. C. Shadden, K. Katija, M. Rosenfeld, J. E. Marsden, and J. O. Dabiri, "Transport and stirring induced by vortex formation," J. Fluid Mech. 593, 315-331 (2007)

${ }^{34}$ B. M. Cetegen and J. P. Aguirre, "Analysis of molecular mixing and chemical reaction in a vortex pair," Phys. Fluids 12, 2211-2216 (1990).

${ }^{35} \mathrm{R}$. Verzicco and P. Orlandi, "Mixedness in the formation of a vortex ring," Phys. Fluids 7, 1513-1515 (1995).

${ }^{36}$ K. B. Southerland, J. R. Porter III, W. J. A. Dahm, and K. A. Buch, "An experimental study of the molecular mixing process in an axisymmetric laminar vortex ring," Phys. Fluids A 5, 1385-1392 (1991).

${ }^{37}$ S. Rajes and K. Mahesh, "Passive scalar mixing in vortex rings," J. Fluid Mech. 582, 449-461 (2007).

${ }^{38}$ A. B. Olcay and P. S. Krueger, "Measurement of ambient fluid entrainment during vortex ring formation," Exp. Fluids 44, 235-247 (2008).

${ }^{39}$ A. B. Olcay and P. S. Krueger, "Momentum evolution of ejected and entrained fluid during laminar vortex ring formation," Theor. Comput. Fluid Dyn. 24, 465-482 (2010).

${ }^{40}$ P. Meunier and E. Villermaux, "The diffusive strip method for scalar mixing in two dimensions," J. Fluid Mech. 662, 134-172 (2010).

${ }^{41}$ J. M. Ottino, "Mixing, chaotic advection, and turbulence," Annu. Rev. Fluid Mech. 22, 207-253 (1990).

${ }^{42}$ E. Villermaux, "Simple ideas on mixing and fragmentation," Chaos 14, 924-932 (2004). 